\title{
FPGA Implementation of a W-CDMA System Based on IP Functions
}

\author{
Aifeng Ren ${ }^{1,2}$, Qinye Yin $^{1}$ \\ 1. School of Electronics and Information Engineering, \\ Xi'an Jiaotong University, Xi'an 710049, China \\ 2. School of Electronic and Engineering, \\ XiDian University, Xi'an 710071, China
}

\begin{abstract}
This paper presents architecture design and FPGA implementation for W-CDMA transceiver system. Altera high-density, high-performance programmable logic devices (PLDs) combined with intellectual property (IP) functions and the Quartus II development software provide a complete solution for the wireless communications application. The paper describes how to implement a wideband code division multiple access system that conforms to the IMT-2000 standard using Altera field programmable gate array (FPGA) devices and IP functions.
\end{abstract}

Key-Words: - IMT-2000, W-CDMA, Rake receiver, FPGA, Intellectual property, interference cancellation.

\section{Introduction}

In the wireless world, the demand for advanced information services is growing. Voice and low-rate data services are insufficient in a world where high-speed internet access is taken for granted. The trend is toward global information networks that offer flexible multimedia information services to users on demand, anywhere, anytime. The need to support bandwidth-intensive multimedia services places new and challenging demands on cellular systems and networks.

The third-generation (3G) mobile communication (International Mobile Telecommunications-2000, IMT-2000) systems are being developed to support a wide range of bearer services with low to high data rates: up to $144 \mathrm{kbps}$ in vehicular, $384 \mathrm{kbps}$ in outdoor low-mobility, and 2Mbps in indoor environments[1]. The data modulation scheme is Quadrature Phase Shift Keying (QPSK) for downlink and Binary Phase Shift Keying (BPSK) for uplink. Hybrid Phase Shift Keying (HPSK) is applied to spreading modulation in uplink. Detection is based on pilot-symbol-aided coherent detection. For downlink, pilot symbols are time-multiplexed, which helps minimize delays in TPC and simplify the reception circuit in the MS. For uplink, pilot symbols are spread by spreading codes different from the data and are I/Q-multiplexed with the data. This ensures continuous transmission even when variable-rate transmission is carried out, and minimizes the peak factor in the transmission waveform[2].

Wideband CDMA (W-CDMA), proposed by 3GPP (Third Generation Partnership Project), is one of the major proposals for ITU IMT-2000 RTT
(Radio Transmission Technology ) standards. In order to provide multimedia services, the scheme must be efficient even when there is a combination of services at various speeds, ranging from high to low data rates. For downlink, a spreading code that assures Orthogonal Variable Spreading Factor (OVSF) is applied, which generates codes that are orthogonal to each other even if the symbol rate (SF, i.e. code length) is different. This enables the provision of various bit rate services through channels that are orthogonal to each other. As for error-correction codes, studies were conducted on the application of turbo codes to mobile communications, which are claimed to have high error-correction performance for relatively high-speed transmissions. Turbo codes are adopted with an optimized interleaver.

The advanced capacity enhancement techniques, which are contemplated in academic circles, require a high throughput platform. A programmable solution that provides flexibility, zero non-recurring engineering (NRE) costs, and the required throughput is the right solution for third-generation wireless applications[3].

Altera high-density, high-performance programmable logic devices (PLDs) combined with intellectual property (IP) functions and the Quartus development software provide a complete solution for the wireless communications market. This application note describes how to implement a wideband code division multiple access (W-CDMA) system that conforms to the IMT-2000 standard using Altera devices and IP functions. 


\section{System Implementation}

\subsection{Transmitter Architecture}

Figure 1 shows the proposed digital architecture of a downlink transmitter that supports the W-CDMA standard.

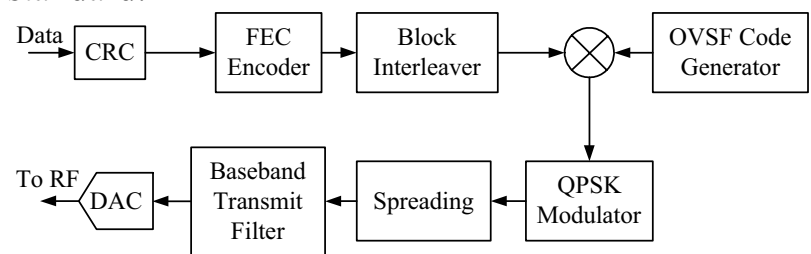

Fig.1 W-CDMA transmitter digital architecture

To conform to the W-CDMA standard, cyclic redundancy code (CRC) bits are added for detecting block errors. This is followed by channel encoding (Forward Error Correction, FEC) and interleaving. By two layers of spreading code sequences, in-phase and quadrature components in the phase plane mapped following data modulation are spread across the spectrum. To transmit a signal within the specified bandwidth, the resulting chip data bits are shaped using a pulse shaping filter. Then, the signal goes through carrier modulation and up-conversion to radio frequency (RF), and is then sent to the antenna to be transmitted over the air.

The various block functions used in the transmitter digital architecture, such as CRC checker, FEC, Block interleaver, OVSF channelization codes, spreading codes and QPSK modulator, are provided in Altera MegaCore functions or IP functions, which can implement these functions and therefore meets the third-generation standard requirements.

\subsubsection{Forward Error Correction}

Turbo encoding gives a relatively large encoding gain which a reasonable computational complexity. This encoding scheme is useful for data services that permit longer transmission delays. The Altera Turbo Encoder/Decoder IP function dramatically shortens design cycles. The Turbo Decoder IP function is a high-performance logarithmic "maximum a posteriori" (max-logMAP) decoder for maximum error correction. Figure 2 shows the block diagram of Altera Turbo Encoder/Decoder IP function.

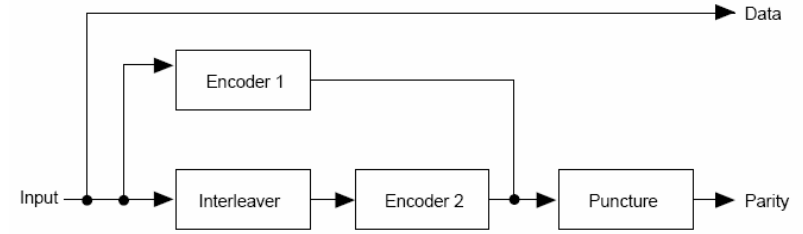

(a) Turbo Encoder block diagram

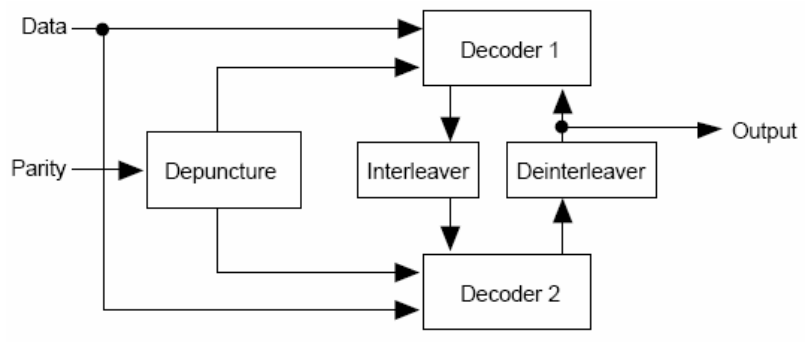

(b) Turbo Decoder block diagram

Fig.2 The block diagram of Turbo Encoder/Decoder IP function

\subsubsection{QPSK Modulator}

Figure 3 shows a modulator that performs baseband filtering and carrier modulation in the digital domain.

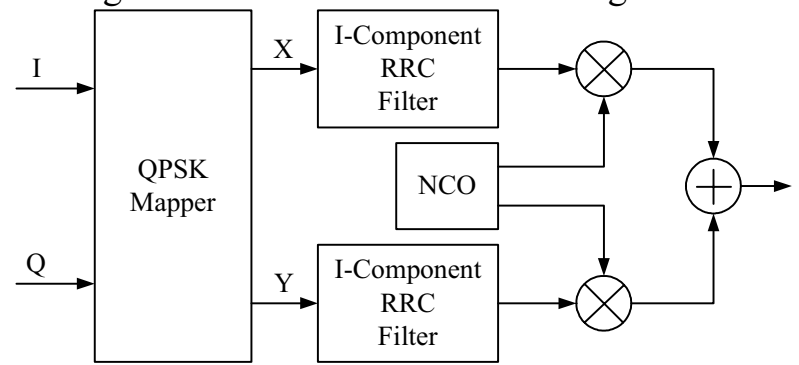

Fig.3 Implementation diagram of QPSK Modulator

Using a digital I/Q modulator instead of an analog one has several advantages, including: (a) Channels can be selected in the digital domain using a numerically controlled oscillator (NCO) and a digital mixer. (b) The direct digital synthesizer gives more precise frequency selection, shorter settling time, good amplitude and phase balance. (c) A digital filter provides extremely linear phase and a very low shape factor.

To meet the W-CDMA requirements, the IP functions can be used to build a complete modulator, such as FIR compiler to create a root raised cosine interpolation filter, $\mathrm{NCO}$ compiler to create an NCO, LPM MULT LPM function to create a digital mixer.

Figure 4 shows the MegaWizard Plug-In of FIR compiler. Here, the FIR filter block is a 60-tap, RRC filter with cut-off frequency of $5 \mathrm{MHz}$, assuming an 80-MSPS sample rate.

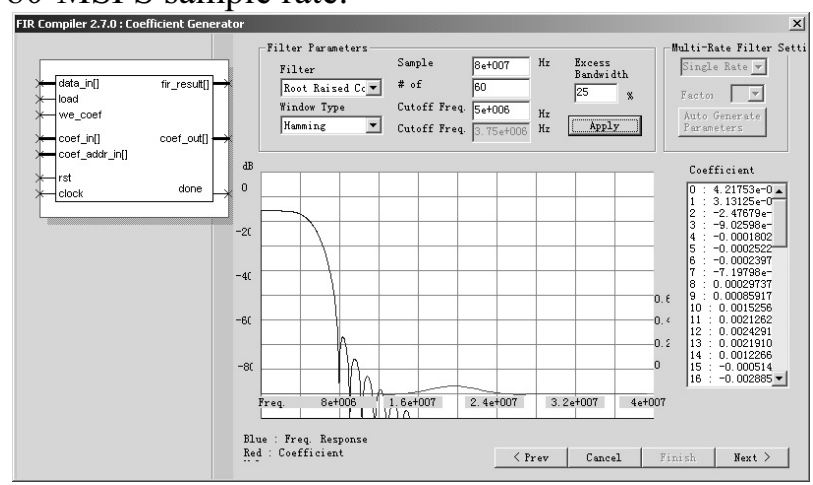

Fig.4 RRC Filter parameters 
Figure 5 shows the MegaWizard Plug-In of NCO compiler. The NCO block, which is $60-\mathrm{MHz}$ bandwidth for channel mapping and high spurious free dynamic range (SFDR), is implemented using the multiplier-based architecture, which reduces memory usage by using the hardware multipliers in the selected FPGA device.

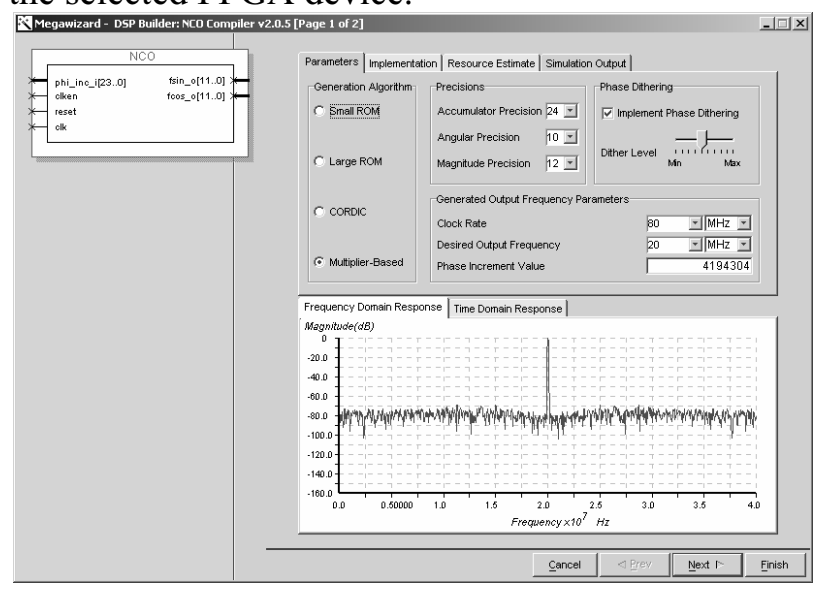

Fig.5 NCO IP parameters

The NCO Compiler can be used to generate a variety of NCO architectures. Using the IP Toolbench driven interface that includes both timeand frequency-domain analysis tools, we can create our custom NCO, which outputs a sinusoidal waveform in two's complement representation. The waveform for the generated sine wave is defined

$$
s(n T)=A \sin \left[2 \pi\left(f_{0}+f_{F M}\right) n T+\phi_{P M}+\phi_{D I T H}\right]
$$

Where $T$ is the operating clock period. $f_{F M}$ is a frequency modulating parameter based on the frequency modulation input value. $\phi_{P M}$ is the phase modulation input value. $\phi_{D I T H}$ is the internal dithering value. $A$ is $2^{N}-1$ where $\mathrm{N}$ is the magnitude precision. $f_{0}$ is the unmodulated output frequency based on the input phase increment value $\phi_{I N C}$, which is determined by

$$
f_{0}=\frac{\phi_{I N C} f_{c l k}}{2^{M}} H z
$$

where $M$ is the accumulator precision (bit) and $f_{c l k}$ is the clock frequency of the IP core in Hz.

Figure 6 shows the proposed functional QPSK modulator design.

\subsection{Receiver Architecture}

Figure 7 shows the proposed digital architecture of a receiver that supports the W-CDMA standard. Each block can be implemented in an FPGA device using IP functions or FPGA-based embedded processor, such as Nios II.

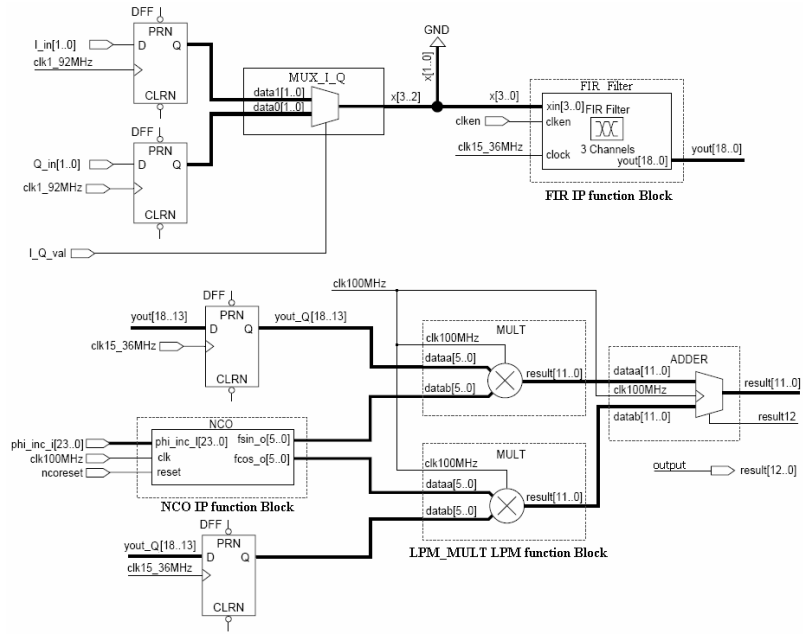

Fig.6 The functional QPSK modulator design

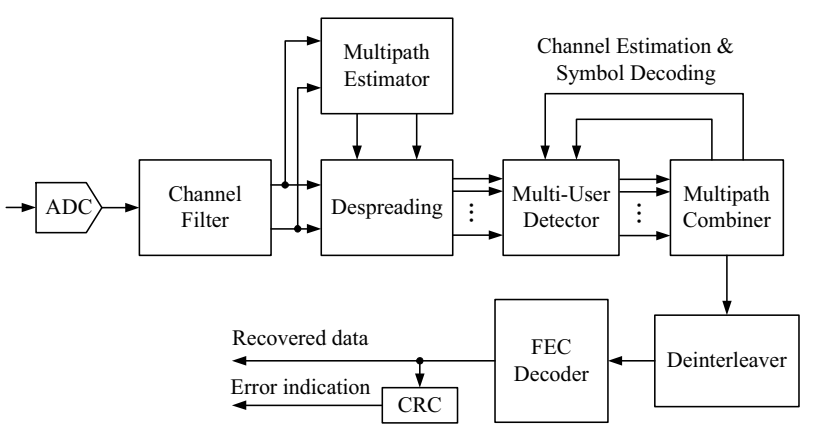

Fig.7 W-CDMA receiver architecture

\subsubsection{Despreading and RAKE receiver}

A signal transmitted through the wireless channel may be severely distorted due to co-channel interference, adjacent channel interference (or multiple access interference), thermal noise, and multipath fading[5]. The most severe distortion comes from fading, which changes the bit error rate (BER) curve from an exponential to a linear curve. To be effective, the system requires a despreader that can simultaneously despread the numerous multipaths of both a single user as well as multiple users. A RAKE receiver, with its multiple fingers to despread different multipaths, is well suited for this function.

A full-matched filter eliminates the need for dedicated delay-locked loops (DLLs) connected to the finger to track the multipath and creates a centrally controlled RAKE receiver that is simpler than a distributed one with DLLs. High-density FPGAs with embedded processor Nios II, such as Cyclone and Stratix, can implement a full-matched filter in programmable logic and control logic by NiosII embedded processor.

\subsubsection{Channel Estimator and Signal Decoder}

Figure 8 shows the implementation diagram of channel estimator and signal decoder. 


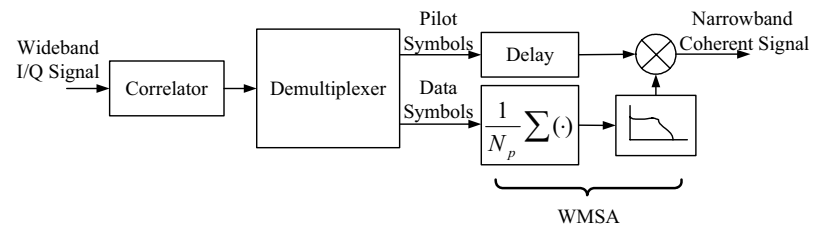

Fig. 8 Channel estimator and signal decoder

This action is part of the RAKE receiver, and is required for coherent detection. One of the schemes used to estimate complex amplitudes is a pilot symbol-assisted channel estimation filter, called a weighted multi-slot averaging (WMSA) channel estimation filter. In this implementation scheme, the wideband signal is converted to a narrowband signal using a binary correlator.

\subsubsection{Multi-User Detector and Interference Cancellation}

W-CDMA is based on direct-sequence (DS) CDMA technology. The capacity of a DS-CDMA system is interference limited. Every user acts as interference for ever other user. The more resistant the system is to interference, the more users can be served. Multi-user detection (MUD) techniques, which are also called joint detection and interference cancellation (IC), reduce the effect of multiple access interference (MAI) and increase system capacity.

Figure 9 shows the categories of MUD algorithm. The maximum likelihood sequence estimator (MLSE) is a well-known optimal detection algorithm. However, it is too complex for practical DS-CDMA systems. Therefore, most research and implementation efforts are focused on developing sub-optimal schemes, which can be classified into linear multi-user detectors and subtractive interference cancellation detectors.

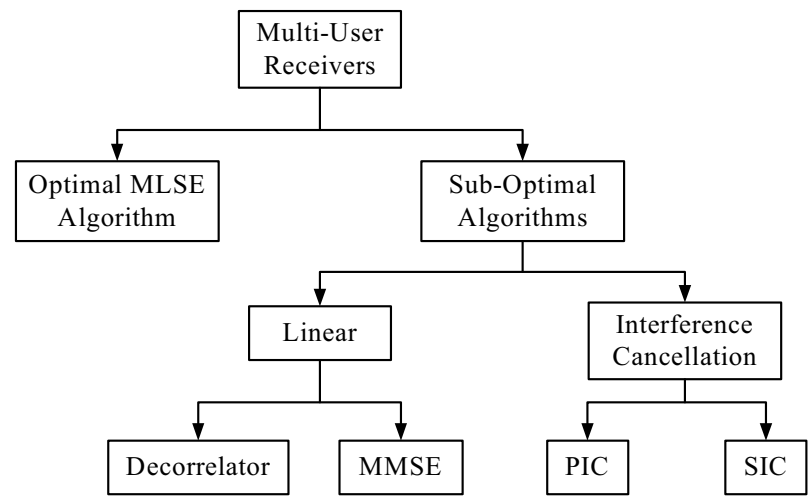

Fig.9 categories of MUD algorithm

The IC scheme can be implemented as a successive IC (SIC) detector and a parallel IC (PIC) detector[6]. The PIC detector separate estimates of the MAI contributed by each user are created in order to subtract out some or all of the MAI seen by the intended user[7].
Figure 10 shows the implementation of one stage of a PIC detector with hard decision for K users.

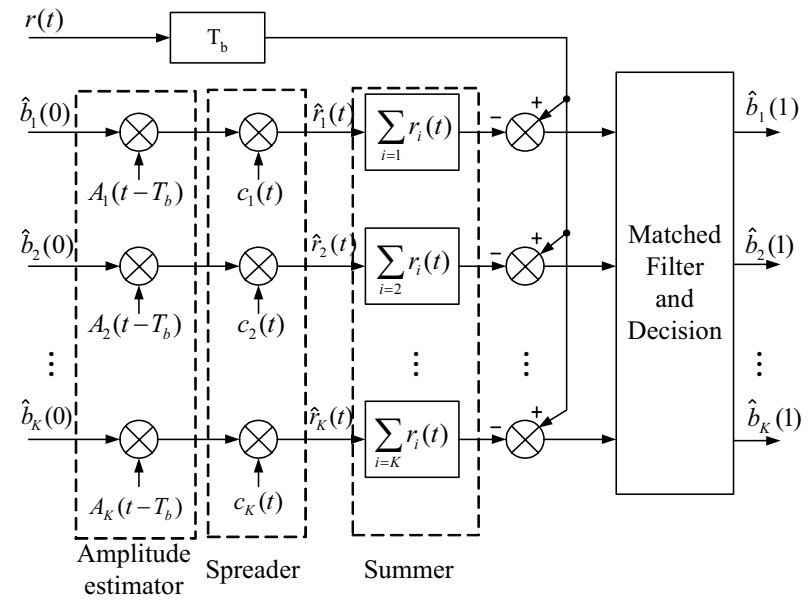

Fig. 10 The implementation of one stage PIC detector

All of the blocks except the matched filter can be implemented in programmable logic. The matched filter, which is complex algorithm for weighted combining and low-speed signal processing requirement, can be implemented effectively in software running in an FPGA-based embedded processor.

\subsubsection{Decoders Implementation}

There are two types of decoders that can be used in the receiver. The Viterbi decoder is used to decode signals encoded using convolutional encoders; the turbo decoder is used with the Turbo encoder.

For Viterbi algorithm, Altera provides the Viterbi IP function that meets W-CDMA requirements.

For Turbo decoder, Altera provides the Turbo decoder IP function, which has the features as Max-logMAP decoder for maximum performance, UMTS-specific interleaver, fully parameterized to tailor decoder to system requirements and memory bank swap mechanism for increased throughput.

\section{Conclusion}

Third generation $(3 \mathrm{G})$ wireless communications systems will offer exciting new functionality such as high quality, high data-rates and a broad range of services at the price of an increased complexity in the design and verification of the $3 \mathrm{G}$ equipments. However, a high throughput platform, such as new system architectures in ASIC, system-on-chip (SoC) and embedded software design, are required to support capacity enhancement techniques. At the same time new design methodologies, based on system level simulation platforms and on the availability of pre-designed IP functions, have to be employed in order to accelerate the development process and to meet the aggressive product development schedules. 
We believe that the proposed architectures serve as a solid foundation for future cost-effective application-specific integrated circuit (ASIC) implementation of the baseband processing circuit in a W-CDMA systems.

\section{References:}

[1] HP Ma, SH Hsu, and TD Chiueh, Design and Implementation of an Uplink Baseband Receiver for Wideband CDMA Communications, IEICE Trans. On Fundamentals of Electronics, Communications and Computer Sciences, Vol.E85-A, No.12, 2002, pp. 2813-2821.

[2] T. Keiji, W-CDMA: Mobile Communications System, John Wiley \& Sons, 2002

[3] Altera AN129, Implementing a W-CDMA System, 9. 2000, Ver.1.0

[4] D. Chris, FPGA Implementation of Carrier Synchronization for QAM Receivers, Journal of VLSI Signal Processing, Vol. 36, 2004, pp.57-71.

[5] G. C. Kim, Maximum Ratio Combining for a WCDMA Rake Receiver, Freescal Semiconductor Application Note. Rev.2, 11, 2004.

[6] S. Ivan, I. P. Klaus, K. E. Troels, M. H. Jack, Implementation aspects for successive interference cancellation in DS/CDMA systems, Wireless Networks, Vol.4, 1998. pp. 447-452.

[7] A. Amr, E. Sald, Implementation of Multi-User Detection for DS-CDMA Communications, NRSC 2005, March 15-17, 2005 Cairo-Egypt.

[8] S. Moshavi, Multi-user detection for DS-CDMA Communications, IEEE Communications Magazine, Vol.34, 8. 1996, pp.124-136. 